\title{
REGULAR CONVERGENCE OF MANIFOLDS WITH BOUNDARY
}

\author{
PAUL A. WhITE
}

In the author's paper [1] it is shown that if a sequence of orientable $n$-dimensional generalized closed manifolds (abbreviated $n$ - $\mathrm{gcm}$ ) converge $(n-1)$-regularly to an $n$-dimensional set, then the limit set is also an orientable $n$-gcm (see Definitions 1 and 2). In this paper a similar result is obtained for manifolds with boundary.

Throughout the paper we assume that our sets are imbedded in a compact Hausdorff space $S$ and that the cycles are Cech cycles with coefficients in an arbitrary field which we will omit from our notation for a cycle. All of the basic homology theory needed is in [7] and a knowledge of it will be assumed. We shall use the notation $\left\{A_{i}\right\} \rightarrow A$ to mean that the sequence of sets $A_{i} C S$ converges to $A \subset S$ as a limit (see p. 10 of [5]). We shall consider convergence only when all of the sets $A_{i}$ are closed, and shall not explicitly state this henceforth; the limit set, as is well known, will always be closed. We shall use small latin letters for points, large ones for sets, and script letters for finite collections of open sets which are coverings of $S$. We shall use " $\cup$ " for point set union or sum, " $\cap$ " for intersection, reserving + and - for the group operations. By $U \wedge A$ we shall mean the subcomplex of the nerve of $U$ whose vertices are elements of the covering $V$ that have a nonvacuous intersection with $A$. If every element of $V$ is contained in some element of $V$, we shall say that $V$ is a refinement of $V$, and shall write $U>V$, or $V=V(V)$; we shall use the notation ${ }^{1} \Pi_{v u}$ to denote a simplicial projection from the nerve of $V$ into the nerve of $\mathcal{V}$. By $U \subset \subset V$ we mean that $\bar{U} \subset V$, and by $U \gg V$ we mean that the closure of the elements of $U$ form a refinement of $\mathcal{V}$, and by $U>* V$, we mean that the stars of the elements of $V$ form a refinement of $\mathcal{V}$. If a cell $\sigma$ of a covering $U$ has a nucleus (the intersection of all the open sets representing the vertices of $\sigma$ ) that meets a set $B$, we say that $\sigma$ is on $B$; if all the cells with nonzero coefficients in a chain $C^{r}$ of $V$ are on $B$, we say that $C^{r}$ is on $B$. If the nucleus of $\sigma$ is in the open set $Q$, we say $\sigma$ is in $Q$. We shall use the symbol " $\partial$ " to denote the boundary operator.

DEFINITION 1. We say that $\left\{A_{i}\right\} \rightarrow A$-regularly if and only if $\left\{A_{i}\right\} \rightarrow A$ and corresponding to any coverings $V, \mathcal{V}$, there is a covering $V_{n}^{\prime}=V_{n}^{\prime}(U)$ and an integer $N$ each depending only on $U$, and a cover-

Received by the editors October 29, 1952.

1 Because of notational difficulties, lower case italic subscripts $u$ and $v$ are used here instead of capital script. 
ing $\boldsymbol{V}_{n}^{\prime}=\mathcal{V}_{n}^{\prime}(\mathcal{U}, \mathcal{V})$ such that if, for $i>N$ and $r \leqq n, Z^{r}\left(\mathcal{V}_{n}^{\prime} \wedge A_{i}\right)$ is a cycle on $A_{i}$ with diameter $\left\langle\bigcup_{n}^{\prime}\right.$, then $\Pi_{v v_{n}} Z^{r}\left(\mathcal{V}_{n}^{\prime} \wedge A_{i}\right)$ bounds a chain on $\mathcal{\wedge} \wedge A_{i}$ with diameter $<U$.

Definition 2. The compact space $M$ will be called an $n$-dimensional generalized manifold $(n-\mathrm{gm})$ with boundary if there exists a closed subset $K$ of $M$ such that

(a) $M=K \cup A$ where $A$ is open and $K=\bar{A}-A$,

(b) $\operatorname{dim} M=n$ (in the sense of Lebesgue, see p. 195 of [7]),

(c) $p_{r}(M \bmod K, x)=0$ for all $x \in M, r \leqq n-1$,

(d) $p_{n}(M \bmod K, x)=1$ for all $x \in M$,

(e) $\operatorname{pr}(M, x)=0$ for all $x \in K, r \leqq n$.

This definition reduces to Wilder's definition of an $n$-dimensional generalized closed manifold ( $n$-gcm) when $K=0$ (see p. 244 of [7]).

We first prove the following theorem which will be useful in proving the main result.

THEOREM 1. If $\left\{A_{i}\right\}$ and $\left\{B_{i}\right\}$ are sequences of closed sets such that $\left\{A_{i}\right\} \rightarrow A$ and $\left\{B_{i}\right\} \rightarrow B$, both n-regularly, and $\left\{A_{i} \cap B_{i}\right\} \rightarrow A$ $\cap B(n-1)$-regularly, then $\left\{A_{i} \cup B_{i}\right\} \rightarrow A \cup B$ n-regularly.

Proof. Let $V, \mathcal{V}$ be arbitrary coverings of the space. Choose $V_{1}>^{*} V$, then there exist $V_{n}^{\prime}>^{*} V_{1}, N_{1}$ and $V_{n}^{\prime \prime}>* V_{1}, N_{2}$; also $\mathcal{V}_{n}^{\prime}$ and $\mathcal{V}_{n}^{\prime \prime}$ in the definition of $n$-regular convergence of $\left\{A_{i}\right\}$ and $\left\{B_{i}\right\}$ respectively corresponding to $U_{1}, \mathcal{V}$. Choose $V_{n}^{\prime \prime \prime}>\left(\mathcal{U}_{n}^{\prime}, \mathcal{V}_{n}^{\prime \prime}\right)$ and $V_{n}^{\prime \prime}>* V_{n}^{\prime \prime \prime}$ and $V_{n}^{\prime \prime \prime}>\left(\mathcal{V}_{n}^{\prime}, V_{n}^{\prime \prime}\right)$. There exists a covering $V_{n-1}^{\prime}$ $>^{*} \mathcal{V}_{n}^{\prime \prime}, N_{3}$, and $\mathcal{V}_{n-1}^{\prime}$ in the definition of the $(n-1)$-regular convergence of $\left\{A_{i} \cap B_{i}\right\}$ corresponding to $\mathcal{V}_{n}^{\prime 0}$ and $\mathcal{V}_{n}^{\prime \prime \prime}$. Also suppose $\mathcal{V}_{n-1}^{\prime}$ is chosen so that any cell of $\mathcal{V}_{n-1}^{\prime}$ on both $A$ and $B$ will also be on $A \cap B$. Choose $\nu^{\prime} \gg V_{n-1}^{\prime}$ and $N_{4}$ according to Theorem 2 of [1] so that $\Pi_{v_{n-1}{ }^{\prime} v^{\prime}}$ maps $C^{r}\left(\mathcal{V}^{\prime} \wedge A_{i}\right)$ and $C^{r}\left(\mathcal{V}^{\prime} \wedge B_{i}\right)$ for $i>N_{4}$ homomorphically into $C^{r}\left(\mathcal{V}_{n-1}^{\prime} \wedge A\right)$ and $C^{r}\left(\mathcal{V}_{n-1}^{\prime} \wedge B\right)$, respectively. Finally choose $N_{5}$ according to Theorem 1 of [1] such that if $i>N_{5}$, then the identity projection maps $C^{r}\left(\mathcal{V}_{n-1}^{\prime} \wedge(A \wedge B)\right)$ isomorphically into $C^{r}\left(\mathcal{V}_{n-1}^{\prime} \wedge\left(A_{i} \wedge B_{i}\right)\right)$. Let $N=N_{1}+N_{2}+N_{3}+N_{4}+N_{5}$. Consider $i>N$ and a cycle $Z^{r}$ on $\nu^{\prime} \wedge\left(A_{i} \cup B_{i}\right)$ with diameter $\left\langle V_{n-1}^{\prime}\right.$. Let $Z^{r}$ $=Z_{1}^{r}+Z_{2}^{r}$ where $Z_{1}^{r}$ is the part of $Z^{r}$ on $A_{i}$ and $Z_{2}^{r}=Z^{r}-Z_{1}^{r}$; hence on $B_{i}$. Let $Z^{r-1}=\partial Z_{1}^{r}=-\partial Z_{2}^{r}$, then $\Pi_{v_{n-1}{ }^{\prime} v^{\prime}} Z^{r-1}$ is on both $A$ and $B$ and, hence, on $A \cap B$. Applying the identity projection, we conclude that $\Pi_{v_{n-1}{ }^{\prime} v^{\prime}} Z^{r-1}$ is on $A_{i} \cap B_{i}$ and, of course, has diameter $<V_{n-1}^{\prime}$. Thus there exists a chain $C^{r}$ on $\mathcal{V}_{n}^{\prime \prime \prime} \wedge\left(A_{i} \wedge B_{i}\right)$ with diameter $<\mathcal{V}_{n}^{\prime \prime}$ such that $\partial C^{r}=\Pi_{v_{n}^{\prime \prime \prime} v_{n-1}} \Pi_{v^{\prime}{ }_{n-1} v^{\prime}} Z_{1}^{r-1}=\Pi_{v_{n}^{\prime \prime \prime} v^{\prime}} Z_{1}^{r-1}$. Now $\Pi_{v_{n}^{\prime \prime \prime} v^{\prime}} Z_{1}^{r}$ $+C^{r}$ and $\Pi_{v_{n}^{\prime \prime \prime} v^{\prime}} Z_{2}^{r}+C^{r}$ are cycles of $\mathcal{V}_{n}^{\prime \prime \prime} \wedge A_{i}$ and $\mathcal{V}_{n}^{\prime \prime \prime} \wedge B_{i}$, respectively, each with diameter $\left\langle\mathcal{V}_{n}^{\prime \prime \prime}\right.$ (in fact each is on the same element of $\left.V_{n}^{\prime \prime \prime}\right)$. Now $\Pi_{v v_{n}{ }^{\prime \prime \prime}}\left(\Pi_{v_{n}{ }^{\prime \prime \prime} v^{\prime}} Z_{1}^{r}-C^{r}\right)$ bounds a chain $C_{1}^{r+1}$ of 
$\nu \wedge A_{i}$ with diameter $\left\langle\bigcup_{1}\right.$ and $\Pi_{v v_{n}{ }^{\prime \prime}}\left(\Pi_{v_{n}{ }^{\prime \prime \prime} v^{\prime}} Z_{2}^{r}+C^{r}\right)$ bounds a chain $C_{2}^{r+1}$ of $\mathcal{Y} \wedge B_{i}$ with diameter $\left\langle\mathcal{V}_{1}\right.$. Now each of the above cycles is on an element $U_{n}^{\prime \prime \prime} \in U_{n}^{\prime \prime \prime}$; hence in $\operatorname{St}\left(U_{n}^{\prime \prime \prime}, V_{n}^{\prime \prime \prime}\right)$ Can element $U_{1} \in V_{1}$. Since each bounds a chain on some element of $V_{1}$ which must certainly meet $U_{1}$, it follows that both chains $C_{1}^{\text {r+1 }}$ and $C_{2}^{r+1}$ are on $\operatorname{St}\left(U_{1}, V_{1}\right) \subset$ some element $U \in \mathcal{V}$. Thus by adding we obtain $\Pi_{v v^{\prime}} Z_{1}^{r}-\Pi_{v v_{n}}{ }^{\prime \prime} C^{r}+\Pi_{v v^{\prime}} Z_{2}^{r}+\Pi_{v v^{\prime}}, C^{r}=\Pi_{v v^{\prime}} Z^{r}$ bounds $C_{1}^{r+1}+C_{2}^{r+1}$, which is a chain on $\mathcal{V} \wedge\left(A_{i} \cup B_{i}\right)$ with diameter $<U$. Thus $\left\{A_{i} \cup B_{i}\right\} \rightarrow n$-regularly to $A \cup B$.

THEOREM 2. If $\left\{M_{i}\right\}$ is a sequence of orientable generalized n-manifolds with boundaries $\left\{K_{i}\right\}$, such that $\left\{M_{i}\right\} \rightarrow M(n-1)$-regularly and $\left\{K_{i}\right\} \rightarrow K(n-2)$-regularly, then $M$ is an orientable $n$-gm with boundary $K$.

Proof. Defíne a second sequence of topologically equivalent orientable manifolds $\left\{M_{i}^{\prime}\right\} \rightarrow(n-1)$-regularly to $M^{\prime} \ni M_{i}^{\prime} \cap M_{i}=K_{i}$ and $M^{\prime} \cap M=K$. By Theorem 1, $\left\{C_{i}=M_{i} \cup M_{i}^{\prime}\right\} \rightarrow(n-1)$-regularly to $C=M \cup M^{\prime}$. Also by results in paper [2], $C_{i}$ is a closed orientable $n$-gm for each $i$ and by results in the paper [1] we have $C$ is an orientable $n-\mathrm{gcm}$ and $K$ is an orientable $(n-1)-\mathrm{gcm}$. By results in the paper [3], we have $M$ is an orientable $n$-gm with boundary $K$.

This result includes as a special case G. T. Whyburn's Theorem 1 (see [6]) that if a sequence of 2-cells $\left\{Z_{i}\right\} \rightarrow 1$-regularly to $Z$, and have boundary simple closed curves $\left\{J_{i}\right\} \rightarrow 0$-regularly to $J$, then $Z$ is a 2-cell with $J$ as boundary. In fact we can conclude the following more general result concerning any 1-regular convergent sequence of 2 manifolds with boundary.

THEOREM 3. If $\left\{M_{i}\right\}$ is a sequence of connected orientable separable 2-gms with boundaries $\left\{K_{i}\right\}$ (i.e. by Theorem 20 of [4] each $M_{i}$ is a classical orientable 2-manifold from which a finite number of open 2-cells whose closures are disjoint have been deleted and $K_{i}$ consists of the union of the 1-spheres that form the boundaries of the 2-cells) such that $\left\{M_{i}\right\}$ converges 1-regularly to the separable set $M$ and $\left\{K_{i}\right\}$ converges 0 -regularly to $K$, then $M$ is an orientable 2-manifold with boundary $K$ and all but a finite number of the $M_{i}$ are homeomorphic with $M$.

Proof. By the proof of Theorem 2, we can construct a second sequence of homeomorphic 2-manifolds $\left\{M_{i}^{\prime}\right\}$ with the same boundaries $\left\{K_{i}\right\}$ such that $\left\{M_{i}^{\prime}\right\} \rightarrow 1$-regularly to $M^{\prime}$, which is homeomorphic to $M$ and $\ni M \cup M^{\prime}$, is a closed orientable 2-manifold. Since all but a finite number of $M_{i} \cup M_{i}^{\prime}$ must have the same betti numbers as $M \cup M^{\prime}$, it follows that $M_{i} \cup M_{i}^{\prime}$ is homeomorphic to 
$M \cup M^{\prime}$ for all but a finite number of the subscripts. Since $M_{i}$ and $M_{i}^{\prime}$ as well as $M$ and $M^{\prime}$ are homeomorphic, it follows that $M_{i}$ and $M$ are homeomorphic for all but a finite number of $i$ 's.

\section{BIBLIOGRAPHY}

1. P. A. White, Regular convergence in terms of Cech cycles, Ann. of Math. vol. 55 (1952).

2. - On the union of two generalized manifolds, Annali della Scuola Normale Superiore vol. 4 (1950).

3. - Extensions of the Jordan Brouwer separation theorem and its converse, Proc. Amer. Math. Soc. vol. 3 (1952) pp. 488-498.

4. - Some characterizations of generalized manifolds with boundary, Canadian Journal of Mathematics vol. 4 (1952).

5. G. T. Whyburn, Analytic topology, Amer. Math. Soc. Colloquium Publications, vol. $28,1942$.

6. - On sequences and limiting sets, Fund. Math. vol. 25 (1935).

7. R. L. Wilder, Topology of manifolds, Amer. Math. Soc. Colloquium Publications, vol. 32, 1949.

University of Southern California 